\title{
Emergence of Synchronized Beating during the Regrowth of Eukaryotic Flagella
}

\author{
Raymond E. Goldstein, ${ }^{1}$ Marco Polin, ${ }^{1}$ and Idan Tuval ${ }^{2}$ \\ ${ }^{1}$ Department of Applied Mathematics and Theoretical Physics, Centre for Mathematical Sciences, University of Cambridge, \\ Wilberforce Road, Cambridge CB3 OWA, United Kingdom \\ ${ }^{2}$ Department of Global Change Research, Mediterranean Institute for Advanced Studies (CSIC-UIB), E-07190 Esporles, Spain
}

(Received 20 June 2011; published 30 September 2011)

\begin{abstract}
A fundamental issue in the biology of eukaryotic flagella is the origin of synchronized beating observed in tissues and organisms containing multiple flagella. Recent studies of the biflagellate unicellular alga Chlamydomonas reinhardtii provided the first evidence that the interflagellar coupling responsible for synchronization is of hydrodynamic origin. To investigate this mechanism in detail, we study here synchronization in Chlamydomonas as its flagella slowly regrow after mechanically induced self-scission. The duration of synchronized intervals is found to be strongly dependent on flagellar length. Analysis within a stochastic model of coupled phase oscillators is used to extract the length dependence of the interflagellar coupling and the intrinsic beat frequencies of the two flagella. Physical and biological considerations that may explain these results are proposed.
\end{abstract}

DOI: 10.1103/PhysRevLett.107.148103

PACS numbers: 87.16.Qp, 05.45.Xt, 47.63.-b, 87.18.Tt

From unicellular organisms as small as a few microns to the largest vertebrates on Earth, we find groups of beating flagella or cilia that exhibit striking spatiotemporal organization. This may take the form of precise frequency and phase locking, as frequently found in the swimming of green algae [1], or beating with long-wavelength phase modulations known as metachronal waves, seen in ciliates such as Paramecium [2] and in our own respiratory systems. The remarkable similarity in the underlying molecular structure of flagella across the whole eukaryotic world leads naturally to the hypothesis that a similarly universal mechanism might be responsible for synchronization. Although this mechanism is poorly understood, one appealing hypothesis is that it results from hydrodynamic interactions between flagella.

The role of hydrodynamics in synchronization has been the subject of intense theoretical investigation in the half century since the seminal work of Taylor [3] showed that phase synchronization of nearby undulating sheets minimizes viscous dissipation. Although minimizing dissipation is not a general principle from which to derive dynamics, a growing body of work has identified requirements for synchronization within minimal models involving systems of rotating spheres or helices [4-6]. It is now known that oscillators with more than one degree of freedom or those with suitable internal forcing can be synchronized by hydrodynamics [6,7]. Various levels of coordination are also found with more realistic models of flagella [8], which rely necessarily on simplified internal driving of the filaments. Nevertheless, these complex models yield important clues to the generation and regulation of flagellar motion [9].

Experimental progress has lagged behind that of theory, due in large part to the challenge of finding a sufficiently simple model system that can be studied under an appropriately broad set of experimental conditions. But recent work [10] on Chlamydomonas reinhardtii (CR, Fig. 1), a biflagellate green alga well developed as a model to study the biology of the flagellar apparatus [11], suggests that $\mathrm{CR}$ is also ideally suited to study flagellar synchronization. It has a spheroidal body $\sim 5 \mu \mathrm{m}$ in radius and swims by the synchronous breaststrokelike beating at $\sim 60 \mathrm{~Hz}$ of two apical flagella $10-12 \mu \mathrm{m}$ long. Their dynamics can be described by a model of self-sustained phase oscillators, with a coupling that is compatible with hydrodynamic estimates [10]. The high uniformity of flagella lengths in wild-type cells, however, prevented a systematic test of the origin of flagellar coupling.

Here we exploit the ability of CR to shed and slowly regrow its flagella to report the first study of beating dynamics as a function of flagellar length. We find that synchrony develops spontaneously as flagella regrow, with increasing phase-locked periods consistent with an interflagellar coupling that grows linearly with flagellar length.

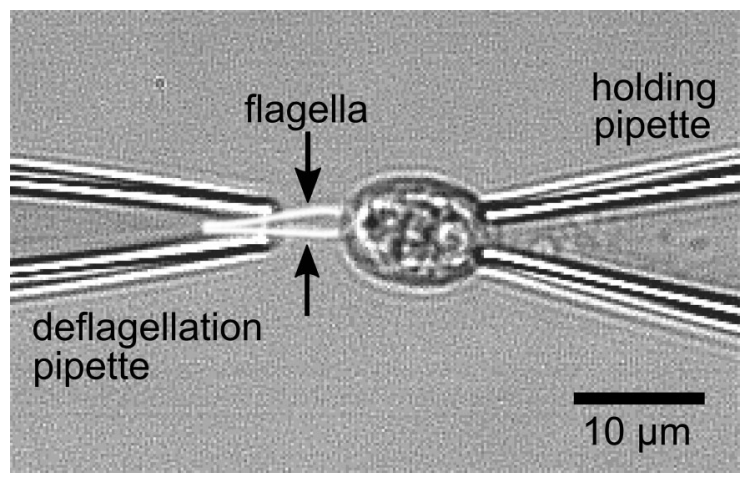

FIG. 1. Setup to induce autotomy. Suction from a holding pipette immobilizes the alga $C$. reinhardtii while a second pipette induces deflagellation by shearing. 
This is compatible with a simple elastohydrodynamic estimate and provides the strongest evidence yet for a mechanical origin of flagellar synchronization.

CR strain CC124 [12] was grown axenically in trisacetate-phosphate medium [11] in a growth chamber (Binder, Germany) set to $24^{\circ} \mathrm{C}$ and a cycle of $16 \mathrm{~h}$ light at $100 \mu \mathrm{Em}^{-2} \mathrm{~s}^{-1}$ (Fluora, OSRAM) and $8 \mathrm{~h}$ dark. Cells from batch cultures in midlog phase $\left(\sim 10^{6}\right.$ cells $\left./ \mathrm{ml}\right)$ were harvested during the light period, and a small aliquot was inserted in a $22 \times 22 \times 3 \mathrm{~mm}$ observation chamber filled with fresh tris-acetate-phosphate medium at room temperature $\left(\sim 22{ }^{\circ} \mathrm{C}\right)$. The chamber was held on a custom stage of a Nikon TE2000-U inverted microscope with a Zeiss $63 \times$ W Plan-Apo water immersion objective (with a numerical aperture of 1.0) fitted with an adapter collar. The objective's working distance of $2.1 \mathrm{~mm}$ allowed imaging cells $\gtrsim 1 \mathrm{~mm}$ from any surface, eliminating wall effects on the motion. Freely swimming cells were captured by applying gentle suction from a manual microinjector (Sutter Instrument Co., USA) to a micropipette with a $2-4 \mu \mathrm{m}$ diameter tip, hosted on a pipette holder (World Precision Instruments, USA) held by a motorized micromanipulator (Patchstar, Scientifica, USA). A custom stage mounted on the holder allowed rotation around the pipette's axis and was used to assess the angle between that axis and the anteroposterior axis of the cell. Cells aligned to $\lesssim 10^{\circ}$ were rotated to bring the flagellar plane into the focal plane of the microscope objective. By shearing the flagella with a second micropipette (Fig. 1), we induced deflagellation, a process actively regulated by the cell; axonemal severing occurs at a precise position just distal to the transition zone inside the flagellar collar, a specialized region of the cell wall [11]. Controlled excision allows the plasma membrane to seal around the axonemal stub, preserving cellular integrity, and is likely a trait already present in the ciliated ancestral eukaryote [11]. Flagella fully regrow over $\sim 2 \mathrm{~h}$ [13] and are fully motile after $\sim 15 \mathrm{~min}$. For each cell we recorded movies $\gtrsim 2$ min long immediately before and then at 20, 30, 40, 50, 60, 70, and 90 min after deflagellation (Fig. 2), using a high-speed video camera (Fastcam SA3, Photron, USA) at $500 \mathrm{fps}$ under bright-field illumination. A long-pass interference filter with a $10 \mathrm{~nm}$ transition ramp centered at $620 \mathrm{~nm}$ (Knight Optical, United Kingdom) prevented any phototactic responses [14]. Movies were processed and analyzed with custom MATLAB routines. After each set of movies, we identified under white light the cis flagellum as the closest to and trans the furthest from the eyespot, a primitive lightsensitive organelle.

Regrowth dynamics of the length $\ell$ averaged over 19 experiments [Fig. 2(a)] can be compared to the balancepoint model of flagellar elongation, which predicts the relation $\ell / \ell_{0}+\ln \left(1-\ell / \ell_{0}\right)=-t / \tau$ [15]. The fit to the data shown in blue yields parameters $\left(\ell_{0}=14.05 \mu \mathrm{m}\right.$ and $\tau=100.9 \mathrm{~min})$ and an overestimate of the early-time data that are both similar to findings in earlier studies $[13,15]$. Interestingly, the length reached after $90 \mathrm{~min}(11.48 \pm$ $0.27 \mu \mathrm{m})$ is significantly longer than that before deflagellation $(10.82 \pm 0.19 \mu \mathrm{m})$. This difference is too large to arise from projection errors caused by the deflagellation protocol. We are unaware of any previous reports of a similar phenomenon under any deflagellation technique employed. This observation supports the idea that flagellar length control is more complex than suggested by the
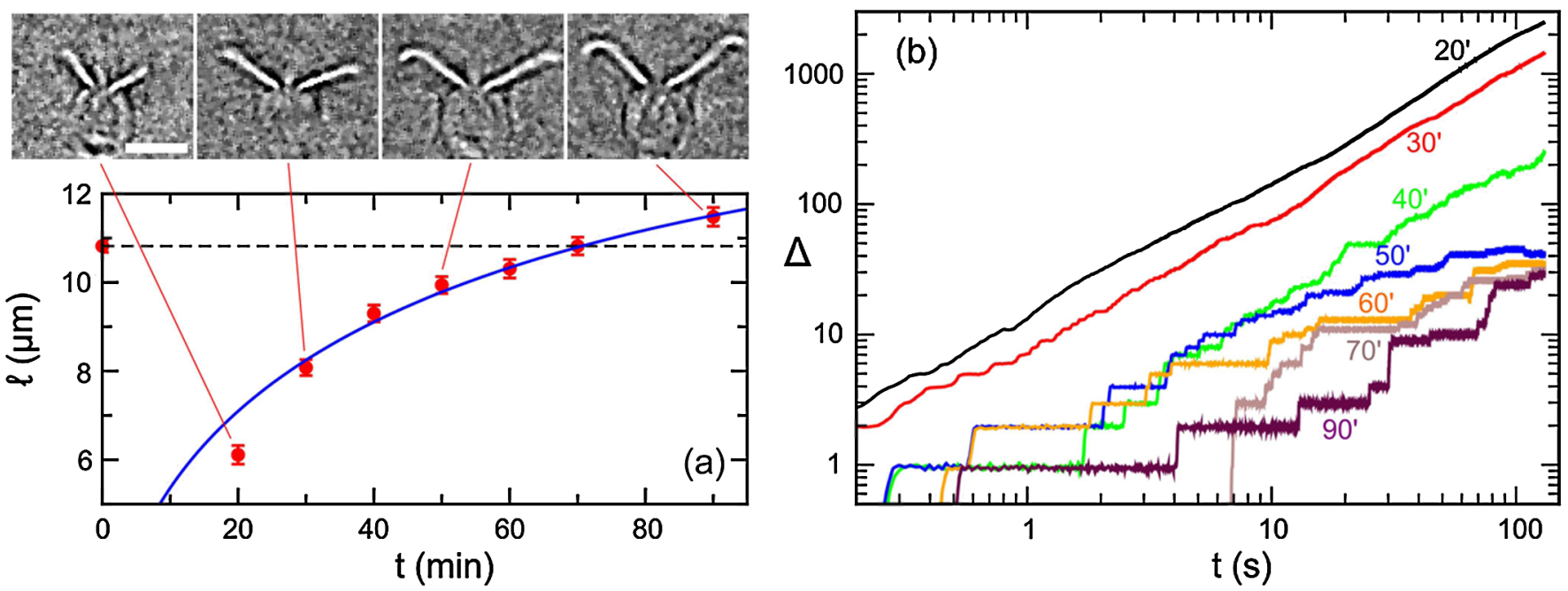

FIG. 2 (color online). Flagellar regrowth and the emergence of synchrony. (a) Regrowth dynamics of flagella after autotomy, and image processed movie frames at the indicated times after deflagellation. The scale bar is $10 \mu \mathrm{m}$. The blue line indicates a fit to the balance-point model of regrowth. (b) Phase difference $\Delta$ as a function of time for a single cell during short experiments at various times (in minutes) after deflagellation, as indicated. Plateaus in $\Delta$, corresponding to periods of synchronous beating, become progressively longer as flagella regrow. 
balance-point model [16]. Indeed, flagella length is sensitive to environmental factors such as the osmolarity of the medium, with effects that can be permanent or transient [17]. Since it has recently been shown that CR flagella possess mechanosensitive channels at their base [18], our observations may reflect different mechanical stress perception in cells held by a micropipette.

We describe the motion of the cis and trans flagella by phase variables $\theta_{\text {cis,trans }}(t)$, which increase by unity at each passage of the corresponding flagellum across a small interrogation region on the side of the cell [10]. The phase difference $\Delta=\theta_{\text {trans }}-\theta_{\text {cis }}$ fluctuates around a constant value during synchrony and drifts during asynchronous beating. Figure 2(b) shows that the dynamics of $\Delta$ depends strongly on flagellar length, with progressively longer periods of synchrony as flagella regrow. To quantify this we employ a model previously shown to describe the dynamics of $\Delta[10]$ :

$$
\dot{\Delta}=\delta \nu-2 \pi \epsilon \sin (2 \pi \Delta)+\xi(t),
$$

where $\delta \nu=\nu_{\text {trans }}-\nu_{\text {cis }}$ is the difference in the intrinsic frequencies of the two flagella, $\epsilon$ is the interflagellar coupling, and the Gaussian white noise $\xi$, satisfying $\langle\xi(t)\rangle=0$ and $\left\langle\xi(t) \xi\left(t^{\prime}\right)\right\rangle=2 T_{\text {eff }} \delta\left(t-t^{\prime}\right)$, represents the naturally occurring variations of flagellar beating.

The parameters $\left(\epsilon, \delta \nu\right.$, and $\left.T_{\text {eff }}\right)$ are determined from the autocorrelation function of $\Delta$ during synchrony and the average duration of a synchronous period. Results from 19 cells are shown in Fig. 3, using the average beat frequency (over all experiments) $\bar{\nu}=63.9 \mathrm{~Hz}$ for nondimensionalization. All parameters vary with flagellar length, with equivalent pre- and postdeflagellation values at similar length. Only $T_{\text {eff }}$ is essentially length-independent (data not shown): Above $\ell=8 \mu \mathrm{m}$ it settles to a constant value $\left[\left\langle T_{\text {eff }} / \bar{\nu}\right\rangle=1.3 \times 10^{-3}\right.$ with a range $\left.(1.0-1.4) \times 10^{-3}\right]$, while it has a minor variation below $\left[\left\langle T_{\text {eff }} / \bar{\nu}\right\rangle=\right.$ $(2.3 \pm 0.4) \times 10^{-3}$ at $\ell=6 \mu \mathrm{m}$ and $(1.6 \pm 0.2) \times 10^{-3}$ at $\ell=8 \mu \mathrm{m}]$. Here the average is over all experiments at a given time after deflagellation. This suggests that flagellar noise is not an intrinsic property of the axoneme but may originate in the unknown mechanism that regulates initiation of beating. Figure 3(a) shows that $\delta \nu / \bar{\nu}$ increases from $10 \%$ to $30 \%$ as $\ell$ increases, with the trans flagellum having always the largest intrinsic frequency. This agrees well with previous studies on cell models and partially deflagellated cells from the same genetic background (CR 137c), which estimated a $\sim 30 \%$ higher beating frequency for full length trans flagella [19]. The intrinsic frequency difference may stem from differential regulation of dynein activity between cis and trans flagella [20], an effect that would tend to increase as $\ell$ increases. As $T_{\text {eff }}$ is nearly constant, this increase in $\delta \nu$ would by itself shorten periods of synchrony, but it is offset by the growth of the coupling $\epsilon / \bar{\nu}$ [Fig. 3(a)], leading to increasing synchronization [Fig. 2(b)].
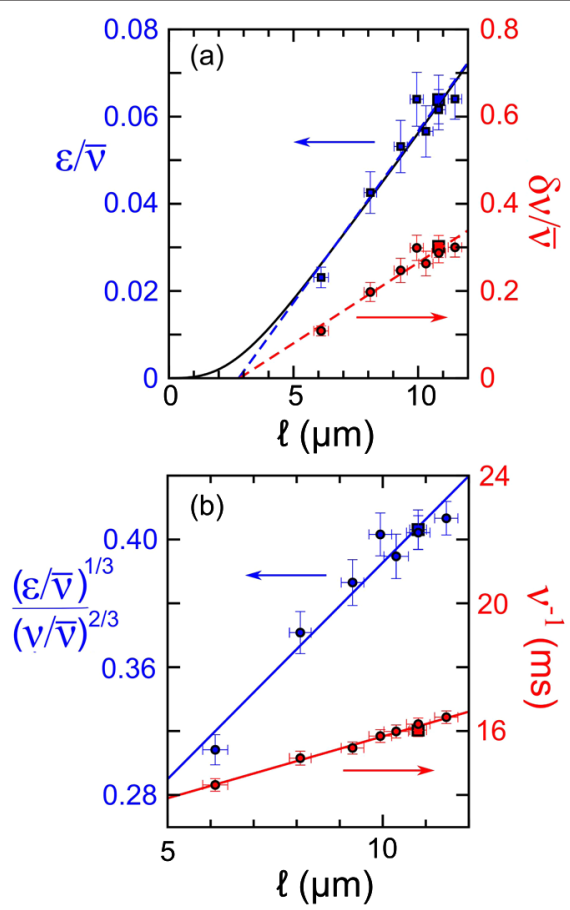

FIG. 3 (color online). Length dependence of parameters in the coupled oscillator model. Large square symbols mark predeflagellation points, while solid circles denote postdeflagellation data. (a) Intrinsic frequency difference and interflagellar coupling as a function of flagellar length $\ell$. Linear fits to these data (dashed lines) both extrapolate to zero at $\sim 3 \mu \mathrm{m}$. The solid black curve indicates a fit to the moving-sphere model with surface proximity effects included. (b) The inverse beat frequency depends linearly on $\ell$. The elastohydrodynamic scaling $\epsilon \sim \nu^{2} \ell^{3}$ is compatible with the observations.

Both $\delta \nu / \bar{\nu}$ and $\epsilon / \bar{\nu}$ display a linear dependence on $\ell$ [Fig. 3(a)] and intercepts with the abscissa at $\ell \sim 3 \mu \mathrm{m}$. Since both parameters are expected to vanish as $\ell \rightarrow 0$, the observed linear trend must change for short flagella. A related study [21] on the length dependence of the propulsive force showed approximately linear growth for $\ell \geq 5 \mu \mathrm{m}$ which also extrapolates to zero at $\ell \sim 3 \mu \mathrm{m}$. The approximate coincidence of the intercept for these three distinct observables suggests a common origin. Short and long flagella may behave differently due to structural inhomogeneities along the flagellum. It is known that in CR the proximal 2-4 $\mu \mathrm{m}$ region of the flagellum has a distinct dynein content [22] and does not contain mastigonemes [23], fine filamentous structures protruding from the flagellar membrane [11]. These differences are likely regulated by intraflagellar transport [24]. Alternatively, the intercept may reflect the need for a minimum load to influence flagellar motion, which is reminiscent of the typical force-speed relation of molecular motors. From previous work [5,10], for $\ell=11 \mu \mathrm{m}$ we expect $\epsilon / \bar{\nu} \sim 0.017$, nearly a factor of 4 lower than observed. This large coupling, consistent with short autocorrelation times for $\Delta(\sim 5$ times shorter than in 
Ref. [10]), may be accounted for by the presence of mastigonemes, which can lead to a drastic increase of the flagellar drag coefficient [23]. Figure 3(b) shows that the beating frequency $\nu$ becomes smaller as flagella elongate in a trend compatible with $\ell^{-1}$, suggestive of a beating mechanism working at constant power output per unit length. The range of $\nu$ values $(15 \%)$ is too small to discount other dependencies or mechanisms, and further experiments are needed to confirm the extent of this behavior.

In a simplified elastohydrodynamic model [5], each flagellum is represented by a sphere moving on a circular orbit through the action of an internal force acting tangential to the circle, and the flagellar elasticity is represented by a Hookean spring which acts to maintain a preferred radius. Two such systems interacting as Stokeslets are found to have a pairwise coupling with the scaling $\epsilon \sim \nu^{2} \ell^{3}$, where the prefactor depends on the fluid viscosity, the flagellar bending rigidity and width, and the density of the flagellar arrangement. Figure 3(b) shows that this scaling is consistent with the data over the accessible range of $\ell$. The linear variation of $\epsilon$ with $\ell$ [Fig. 3(a)] is also consistent with the trend $\nu \sim \ell^{-1}$ described above, but again the extrapolated intercept at $\ell \sim 3 \mu \mathrm{m}$ requires explanation. In the context of this model of moving spheres, one can generalize the hydrodynamic coupling to account for the presence of the cell body by incorporating the known crossover function for Stokeslets near a no-slip wall [25]. From this we obtain the solid curve shown in Fig. 3(a), which displays a linear regime beyond $\ell \simeq 5 \mu \mathrm{m}$ in agreement with the data and a rapid attenuation as $\ell \rightarrow 0$. The lack of data for $\epsilon$ in the regime of small $\ell$ at present makes it difficult to test this mechanism in detail. Moreover, viscous effects from proximity to a no-slip surface do not immediately provide an explanation for the trend in $\delta \nu$.

We have presented the first systematic study of the coupled dynamics of a pair of eukaryotic flagella as a function of their length. Our results show that at compatible flagellar lengths pre- and postdeflagellation dynamics are equivalent, despite the fact that in the latter case flagella are still actively growing. This suggests that the evolution of the coupled motion is governed only by flagellar length and beating frequency and not directly by the internal biochemical state of the cell. The analysis shows that synchrony develops as a result of a growing coupling strength $\epsilon$. In the accessible range of parameters this increase follows a simple elastohydrodynamic form. Taken together, these results give the strongest support available to the hypothesis of a purely mechanical origin of the coupling. These observations also suggest that the coupled dynamics of short $(\Sigma 3 \mu \mathrm{m})$ and long $(\geq 6 \mu \mathrm{m})$ flagella may differ substantially. Exploring this difference in future experiments may lead to important insights into the regulation of flagellar activity.
We are grateful to K.C. Leptos for discussions. This work was supported by an EPSRC Postdoctoral Fellowship (M.P.), the Spanish MICINN "subprograma Ramon y Cajal" (I. T.), the BBSRC, and the European Research Council Advanced Investigator Grant No. 247333 (R.E. G.).

[1] U. Rüffer and W. Nultsch, Cell Motil. Cytoskeleton 7, 87 (1987).

[2] T. L. Jahn and J. J. Votta, Annu. Rev. Fluid Mech. 4, 93 (1972).

[3] G. I. Taylor, Proc. R. Soc. A 209, 447 (1951).

[4] M. Reichert and H. Stark, Eur. Phys. J. E 17, 493 (2005); A. Vilfan and F. Julicher, Phys. Rev. Lett. 96, 058102 (2006).

[5] T. Niedermayer, B. Eckhardt, and P. Lenz, Chaos 18, 037128 (2008).

[6] G. J. Elfring and E. Lauga, Phys. Rev. Lett. 103, 088101 (2009); B. Qian, H. Jiang, D. A. Gagnon, K. S.Breuer, and T. R. Powers, Phys. Rev. E 80, 061919 (2009); J. Kotar, M. Leoni, B. Bassetti, M. Cosentino-Lagomarsino, and P. Cicuta, Proc. Natl. Acad. Sci. U.S.A. 107, 7669 (2010); N. Uchida and R. Golestanian, Phys. Rev. Lett. 104, 178103 (2010); 106, 058104 (2011); R. Golestanian, J. Yeomans, and N. Uchida, Soft Matter 7, 3074 (2011).

[7] P. Lenz and A. Ryskin, Phys. Biol. 3, 285 (2006).

[8] S. Gueron, K. Levit-Gurevich, N. Liron, and J. J. Blum, Proc. Natl. Acad. Sci. U.S.A. 94, 6001 (1997); B. Guirao and J.F. Joanny, Biophys. J. 92, 1900 (2007); Y. Yang, J. Elgeti, and G. Gompper, Phys. Rev. E 78, 061903 (2008).

[9] I. H. Riedel-Kruse, A. Hilfinger, J. Howard, and F. Jülicher, HFSP J. 1, 192 (2007).

[10] M. Polin, I. Tuval, K. Drescher, J. P. Gollub, and R. E. Goldstein, Science 325, 487 (2009); R. E. Goldstein, M. Polin, and I. Tuval, Phys. Rev. Lett. 103, 168103 (2009).

[11] E.H. Harris, The Chlamydomonas Sourcebook (Academic, Oxford, 2009), Vols. 1 and 3.

[12] The Chlamydomonas Resource Center at the University of Minnesota, http://www.chlamy.org.

[13] P. A. Lefebvre, S. A. Nordstrom, J. E. Moulder, and J. L. Rosenbaum, J. Cell Biol. 78, 8 (1978); P. A. Lefebvre, C. M. Asleson, and L. Tam, Semin. Dev. Biol. 6, 317 (1995).

[14] O. A. Sineschekov, K.-H. Jung, and J. L. Spudich, Proc. Natl. Acad. Sci. U.S.A. 99, 8689 (2002); P. Lariguet and C. Dunand, J. Mol. Evol. 61, 559 (2005).

[15] W. Marshall, H. Qin, R. Brenni, J. L. Rosenbaum, Mol. Biol. Cell 16, 270 (2005).

[16] N. Wilson, J. Iyer, J. Buchheim, W. Meek, Semin. Cell. Dev. Biol. 19, 494 (2008).

[17] K. Solter, A. Gibor, Nature (London) 275, 651 (1978).

[18] K. Fujiu, Y. Nakayama, H. Iida, M. Sokabe, and K. Yoshimura, Nat. Cell Biol. 13, 630 (2011).

[19] R. Kamiya and E. Hasegawa, Exp. Cell Res. 173, 299 (1987). 
[20] S. Takada and R. Kamiya, Cell Motil. Cytoskeleton 36, 68 (1997).

[21] R. P. McCord, J. N. Yukich, and K. K. Bernd, Cell Motil. Cytoskeleton 61, 137 (2005).

[22] T. Yagi, K. Uematsu, Z. Liu, and R. Kamiya, J. Cell Sci. 122, 1306 (2009).
[23] S. Nakamura, G. Tanaka, T. Maeda, R. Kamiya, T. Matsunaga, and O. Nikaido, J. Cell Sci. 109, 57 (1996).

[24] M. A. Silverman and M. R. Leroux, Trends Cell Biol. 19, 306 (2009).

[25] J.R. Blake, Proc. Cambridge Philos. Soc. 70, 303 (1971). 